\title{
Donning the powered air-purifying respirator in an emergency obstetric setting amid the COVID-19 outbreak in Singapore: a balance between safety and urgency
}

Singapore Med J 2021; 62(4): 207 https://doi.org/10.11622/smedj.2020099

Dear Sir,

As frontline staff working in the largest maternity unit in Singapore during the COVID-19 outbreak, we wish to raise awareness of the dilemma faced during emergency obstetric procedures that are time-sensitive and crucial in determining obstetric and neonatal outcomes.

During the COVID-19 outbreak, healthcare workers (HCWs) are required to don the powered air-purifying respirator (PAPR) as part of the personal protective equipment (PPE) when attending to suspected or confirmed COVID-19 patients during aerosol-generating procedures, including labour and Caesarean sections. Multiple steps are required when donning the PPE and PAPR, which include turning on the air filter, checking its integrity, adjusting the waist belt and attaching the air filter to the belt, and finally wearing the hood over the head. The entire process takes a considerable amount of time depending on level of training and familiarity with the procedure. While this is a necessary step for the safety of HCWs during the outbreak to prevent disease transmission, in an obstetric setting, where immediate emergency Caesarean sections may be indicated for non-reassuring fetal heart tracings, placental abruption or cord prolapse, every minute of delay affects the prognosis of the mother and fetus. How can we then balance the safety of HCWs with this time urgency in emergency obstetric procedures?

Based on personal experience and that of our colleagues, we have identified potential sources of delay in the COVID-19 obstetric patient-care workflow. Firstly, the anaesthetist and operating theatre staff have to be informed in advance to enable them to prepare a dedicated isolation operating theatre. Secondly, the pathway between the delivery suite and the operating theatre must be cordoned off to prevent other patients or visitors from blocking the way or being at risk of transmission. Finally, HCWs involved have to don the full PPE and PAPR for the procedure. This process involves a multidisciplinary team, including obstetricians, anaesthetists, neonatologists, midwives, and labour ward and operating theatre nurses.

We herein present a few suggestions to address the dilemma of balancing the safety of HCWs with the time-critical nature of emergency obstetric procedures. For one, we recommend that the multidisciplinary team involved should be trained to be familiar with the amended emergency obstetric workflow and the donning of PPE/PAPR. Mock training sessions should be conducted to ensure a more efficient process of patient transfer into the operating theatre once a decision for emergency Caesarean section has been made. Repeated training sessions will decrease the time taken by HCWs to don the PAPR.

Another strategy is early anticipation and having a low threshold when deciding on emergency Caesarean sections for suspected or confirmed COVID-19 obstetric patients. For example, anticipating the need for an emergency Caesarean section when the cardiotocographic (CTG) trace is suspicious and pre-empting the operating theatre team of the potential emergency would provide more time for staff to prepare. This is opposed to deciding on it only when the CTG tracing becomes pathological, at which time the timing of delivery becomes more critical. While this may place an additional strain on resources, such as preparing for a Caesarean section that may potentially not take place, it is a good compromise to help shorten the time to delivery if the need for an emergency Caesarean section arises for suspected or confirmed COVID-19 cases. These measures aim to reduce any compromise to obstetric outcomes in the emergency setting, while at the same time avoiding a trade-off with the safety of the HCWs involved.

By sharing our experience and suggestions, we hope to raise awareness of this dilemma in the emergency obstetric setting during the current COVID-19 outbreak and also allow colleagues from other disciplines involved in emergency care to relate to and learn from our experience.

Yours sincerely,

Krystal Miao Lin $\underline{K o h}^{1}$, Manisha Mathur

1Department of Obstetrics and Gynaecology, KK Women's and Children's Hospital, Singapore. krystalgermaine@gmail.com 